\title{
Transient response in doped germanium photoconductors under very low background operation
}

\author{
S. E. Church, M. C. Price, N. M. Haegel, M. J. Griffin, and P. A. R. Ade
}

\begin{abstract}
Doped germanium photoconductors are the most sensitive detectors for astronomy in the wavelength range $40-240 \mu \mathrm{m}$. Under the extremely low background conditions encountered in cooled satellite instruments, these devices exhibit a number of transient effects, such as slow relaxation after a step change in illumination or bias, and spontaneous spiking at high signal levels. Such behavior can degrade the excellent instantaneous sensitivity of these detectors and create calibration uncertainties. These effects have been observed in the Ge:Be photoconductors and the stressed and unstressed Ge:Ga photoconductors in the Long Wavelength Spectrometer, one of the instruments on the Infrared Space Observatory. A systematic investigation of the transient response of the Long Wavelength Spectrometer detectors to a step change in illumination as a function of operating temperature, bias electric field, and illumination step size has been carried out to determine operating conditions that minimize the effects of this behavior. The transient effects appear to be due primarily to carrier sweep out, but they are not fully explained by existing models for transient response. ๑ 1996 Optical Society of America
\end{abstract}

\section{Introduction}

Doped germanium photoconductors find important applications in astronomy, including satellite-borne instruments in which the photon background is very low. These devices have excellent instantaneous sensitivity, making them the best detectors for the far-infrared (FIR) 40-240- $\mu \mathrm{m}$ wavelength range. However, under certain conditions both doped Ge and doped Si photoconductors exhibit nonlinear behavior that can degrade sensitivity and cause calibration problems. This behavior can be divided into two types: (a) increased noise caused by spontaneous current spiking or current oscillations, ${ }^{1,2}$ and (b) slow response times to changes in illumination, ranging from tens of seconds for germanium devices $^{3,4}$ to tens of minutes for silicon. ${ }^{5,6}$ A careful

\footnotetext{
When this research was done S. E. Church, M. C. Price, M. J. Griffin, and P. A. R. Ade were with the Department of Physics, Queen Mary and Westfield College, Mile End Road, London E1 4NS, U.K. S. E. Church is now with the Division of Physics, Mathematics and Astronomy, California Institute of Technology, MS 59-33, Pasadena, California 91106. N. M. Haegel is with the Department of Physics, Fairfield University, Fairfield, Connecticut 06430 .

Received 9 August 1995; revised manuscript received 18 October 1995.

0003-6935/96/101597-08\$06.00/0

(1) 1996 Optical Society of America
}

characterization of these effects is necessary to minimize and correct for nonideal behavior if maximum sensitivity is to be achieved.

\section{A. Long Wavelength Spectrometer}

The Long Wavelength Spectrometer (LWS) carries out medium resolution $(\lambda / \Delta \lambda \sim 200)$ and highresolution $(\lambda / \Delta \lambda \sim 10000)$ spectroscopy over the wavelength range $45-200 \mu \mathrm{m}$ and is one of four focal plane instruments on the Infrared Space Observatory. Ten $1 \mathrm{~mm}^{3}$ doped germanium photoconductors, manufactured by the Battelle-Institut ${ }^{7}$ and each sensitive to a different spectral range, comprise the LWS detector array. A Ge:Be detector is used for 45-50 $\mu \mathrm{m}$, five unstressed Ge:Ga detectors are used for 50-110 $\mu \mathrm{m}$, and four stressed Ge:Ga detectors are used for 110-200 $\mu \mathrm{m}$ spectral coverage. The stress applied to the long wavelength detectors is tailored to maximize the spectral response for the appropriate wavelength range (in the remainder of this paper, the letters $s$ and $u$ in parentheses distinguish between stressed and unstressed Ge:Ga, respectively). The doping of the Ge:Be detectors is $8 \times 10^{14} \mathrm{Be} \mathrm{cm}^{-3}$ with other dopants below $10^{13} \mathrm{~cm}^{-3}$; the Ge:Ga detectors have a Ga concentration of $2 \times 10^{14} \mathrm{~cm}^{-3}$. The compensation ratio (defined here as the ratio of donor to acceptor concentration) from residual impurities is $\sim 0.01$ for all the detectors. ${ }^{8}$ 
The LWS uses five FIR illuminators ${ }^{9}$ to monitor changes in detector responsivity as a function of time. Each illuminator consists of a Nichrome film on a sapphire substrate. By passing a current through the film, the device can be heated to temperatures up to $150 \mathrm{~K}$. Absolute calibration of the LWS will be performed by regular observations of astronomical sources. Measurements of the performance characteristics of the LWS detectors are described elsewhere ${ }^{10}$ and summarized in Table 1. Excellent instantaneous noise equivalent powers (NEP's) of $\leq 10^{-17} \mathrm{~W} \mathrm{~Hz}^{-1 / 2}$ for the Ge:Be and Ge:Ga (u) detectors, and $<2.0 \times 10^{-18} \mathrm{~W} \mathrm{~Hz}^{-1 / 2}$ for the $\mathrm{Ge}: \mathrm{Ga}(s)$ detectors are achieved under very low background conditions.

\section{B. Nonideal Behavior in the Long Wavelength Spectrometer Detectors}

The slow response time of doped Ge photoconductors to step changes in photon flux influences the calibration procedure for the LWS using the on-board illuminators. Figure 1 shows the response of each LWS detector type to pulses (10 s on, $10 \mathrm{~s}$ off) of infrared illumination at three different illuminator current settings. Even at comparable illumination levels, there are striking differences between the responses of the three detector types. Each individual pulse shows a slow settling time, and at the highest current setting, $10 \mathrm{~s}$ is insufficient for any of the detectors to reach a stable current value before the illuminator is switched off. In contrast, the settling time of the Ge:Ga detectors after the illuminator is switched off is very rapid. The Ge:Be detector, however, shows a long settling time ( $\gg 10 \mathrm{~s})$ after the illuminator is switched off. In all cases a noticeable envelope function is associated with each pulse set, indicating that, even when the illuminator is switched off, the detectors retain a memory of their previous illumination history. It seems that the response characteristics of all the detectors are improved if smaller photon flux steps are used.

Figure 2 shows the response of an LWS Ge:Ga $(u)$ detector to a step change in illumination at six different detector temperatures (although the six response curves are shown on the same figure for clarity, several minutes elapsed between each illuminator step to allow the temperature to be changed). At temperatures $\leq 2.25 \mathrm{~K}$, spontaneous spiking greatly reduces the sensitivity of the detector. This occurs when the detector is operated close to the breakdown field strength, thus permitting impact ionization to cause sporadic breakdown in different parts of the crystal. Spontaneous spiking at temperatures $<2.5 \mathrm{~K}$ is seen in all LWS Ge:Be and Ge:Ga $(u)$ detectors. Each spike is characterized by a fast rise time and short duration $(<10 \mathrm{~ms})$, although the occurrence of a spike often causes a change in responsivity that lasts for several tens of seconds (see Fig. 2). When the sharp spikes are removed from the data, a slow oscillation in the steady current value is often present. Effects such as these severely limit the usable bias range of the unstressed detectors at low operating temperatures. For this reason, the $\mathrm{Ge}: \mathrm{Ga}(u)$ detectors and the $\mathrm{Ge}: \mathrm{Be}$ detector are operated at temperatures near $3 \mathrm{~K}$, despite the unwelcome increase in the dark current at this temperature. ${ }^{10}$ Unstable behavior of this kind is not seen in the Ge:Ga(s) detectors, even at temperatures as low as $1.5 \mathrm{~K}$. Consequently, the stressed detectors are operated at $1.85 \mathrm{~K}$ in the LWS to ensure low dark current and high sensitivity.

This paper describes a systematic investigation of the transient response to a step illumination change of the three types of LWS detectors as a function of operating temperature, illumination step size, and bias electric field, carried out prior to integration of the detectors into the LWS. Optimum operating conditions for the LWS detectors in flight were determined from these data. In Section 4 the results are considered in terms of existing models for transient response in extrinsic photoconductors.

\section{Experimental Arrangement}

A schematic of the components in the ${ }^{4} \mathrm{He}$ dewar used for these tests is shown in Fig. 3. The coldplate temperature can be set to a value between 1.5 and $4.2 \mathrm{~K}$ by adjustment of the vapor pressure above the ${ }^{4} \mathrm{He}$ bath. The detector filter stack contains a metal-mesh narrow-band filter and a short wavelength edge to define a spectral passband with 40-70\% in-band transmission and excellent out-ofband rejection. An illuminator, identical to those in the LWS, is used as the stepped FIR source. High illuminator temperatures $(\sim 150 \mathrm{~K})$ are used to ensure that the filter passband is in, or close to, the Rayleigh-Jeans part of the spectrum, thus reducing the sensitivity of the system to fluctuations in illuminator drive current. Neutral-density filtering attenuates the illuminator signal to levels that will be used in flight. With this arrangement, signal power levels at the detector can be varied from $10^{-17}$ to $10^{-14} \mathrm{~W}$. So that the photon background is mini-

Table 1. Measured Performance Parameters of the LWS Detectors

\begin{tabular}{ccccc}
\hline $\begin{array}{c}\text { Detector } \\
\text { Type }\end{array}$ & $\begin{array}{c}\text { Operating } \\
\text { Temperature }(\mathrm{K})\end{array}$ & $\begin{array}{c}\text { Bias Electric } \\
\text { Field }\left(\mathrm{V} \mathrm{cm} \mathbf{c m}^{-1}\right)\end{array}$ & $\begin{array}{c}\text { Dark Current } \\
(\mathrm{e} / \mathrm{s})\end{array}$ & $\begin{array}{c}\text { Responsivity } \\
(\mathrm{A} / \mathrm{W})\end{array}$ \\
\hline $\mathrm{Ge}: \mathrm{Be}$ & 3.0 & 6.0 & $200-700$ & 0.4 \\
$\mathrm{Ge}: \mathrm{Ga}(u)$ & 3.0 & 2.0 & $250-600$ & $0.4-1.2$ \\
$\mathrm{Ge}: \mathrm{Ga}(s)$ & 1.85 & 0.7 & $50-2100^{a}$ & $2-9^{a}$ \\
\hline
\end{tabular}

${ }^{a}$ The large range quoted for the responsivities and dark currents of the stressed Ge:Ga detectors reflects the differing amounts of stress applied to each element. Increasing the stress increases the dark current and the responsivity. 


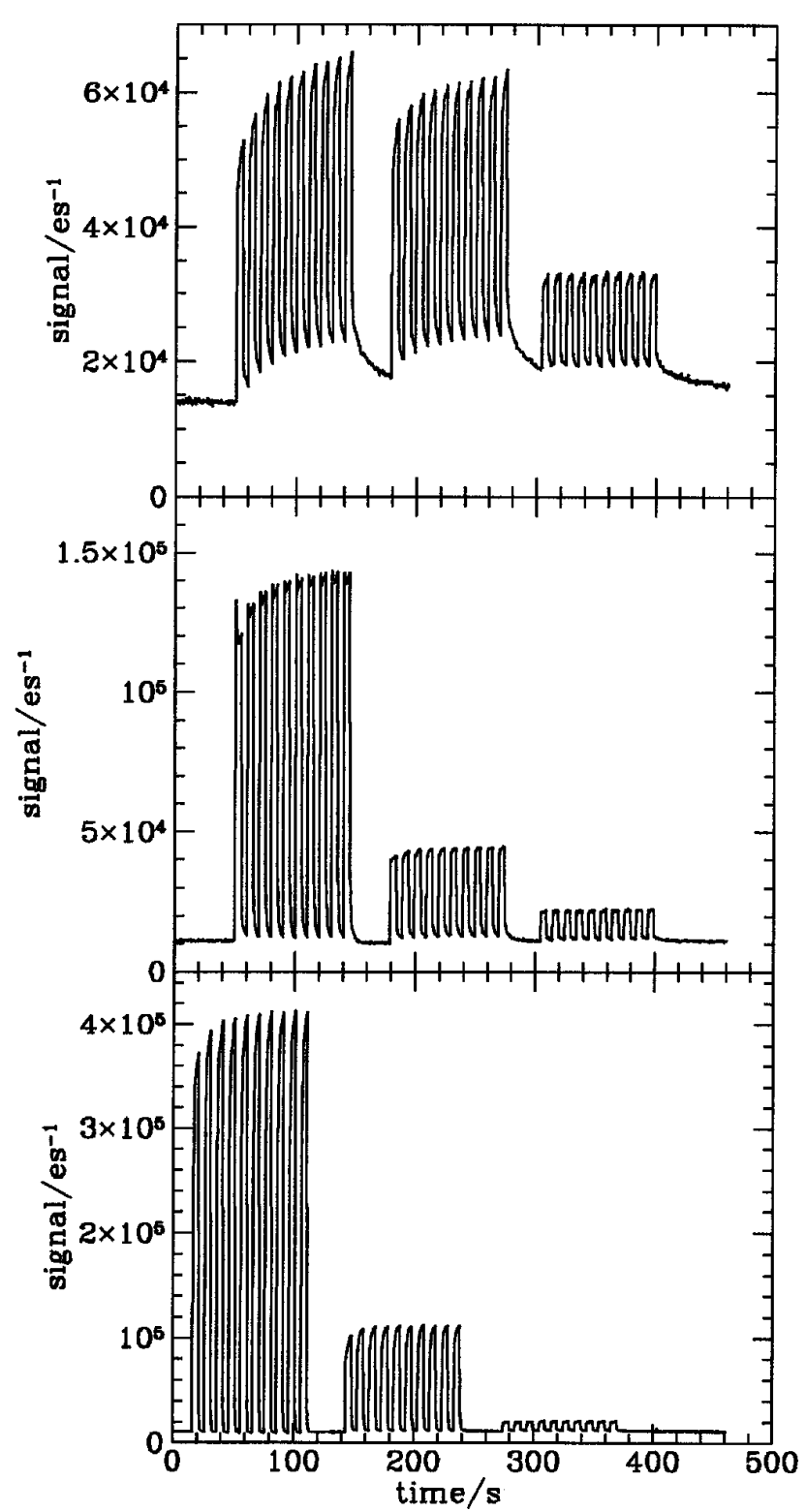

Fig. 1. Transient response of the three LWS detector types to rapid flashes (10 s on, $10 \mathrm{~s}$ off $)$ of a FIR illuminator. The upper, middle, and lower panels show the response of Ge:Be, Ge:Ga $(u)$, and stressed Ge:Ga $(s)$, respectively. The three sets of pulses in each panel correspond to different illumination levels; the first pulse set in each panel corresponds to an illumination level of $\sim 4 \times 10^{7}$ photons $\mathrm{s}^{-1}$.

mized when the illuminator is switched off, the external window of the dewar is sealed with a blanking plate and the cold components are completely surrounded by a blackened radiation shield bolted to the ${ }^{4} \mathrm{He}$ cold plate. When the illuminator is off, the detector current is very close to the measured dark current.

The detector is connected to an integrating amplifier. ${ }^{11}$ During an integration, the 7.5-pF input capacitance of the amplifier is charged up by the detector current. The integrating node is periodically discharged by application of a reset pulse to the device. For reset intervals very much less than the

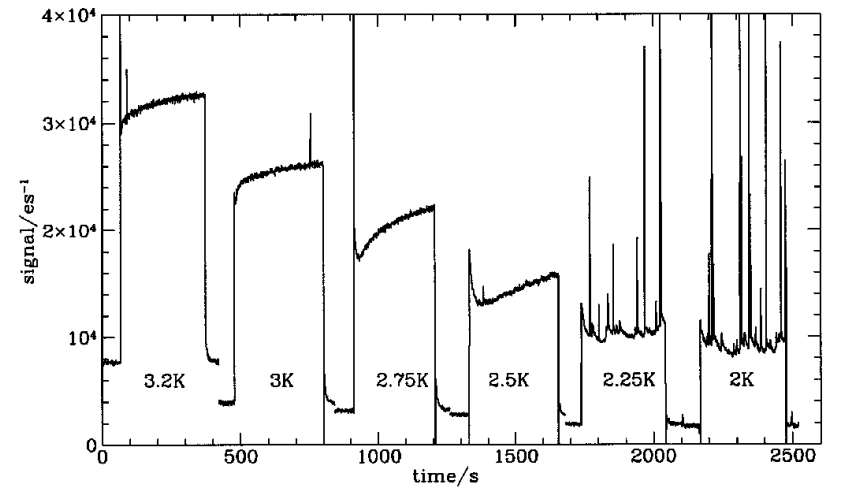

Fig. 2. Transient response to a step change in illumination as a function of operating temperature, observed in a $\mathrm{Ge}: \mathrm{Ga}(u)$ detector at $85 \mu \mathrm{m}$ and $2 \mathrm{~V} \mathrm{~cm}^{-1}$ bias. The five response curves corresponding to different detector operating temperatures are shown as contiguous in time but were actually separated by $10 \mathrm{~min}$ to allow the detector operating temperature to be changed.

RC time constant of the detector-amplifier combination, the output voltage is a linear ramp with time, from which the detector current can be derived. The period between resets is chosen from the range $0.2-4 \mathrm{~s}$, depending on the detector current. The first $0.1 \mathrm{~s}$ of each ramp is corrupted by settling effects associated with the resetting process and is discarded. The illuminator has a known settling time to a current change of $\sim 1 \mathrm{~s}$; therefore, for transient response measurements, the first second of data after an illuminator current change is discarded and detector transient response times $<0.5 \mathrm{~s}$ cannot be accurately measured.

\section{Measurements of Transient Response Times in a Range of Ge Detectors}

A standard procedure was followed to ensure repeatable conditions between illuminator pulses: (a) the detector was allowed to settle in dark conditions for $10 \mathrm{~min}$; (b) 2 min of dark data were collected; (c) the illuminator was switched on for approximately 5 min; (d) after the illuminator was switched off, data acquisition continued for $2-5 \mathrm{~min}$ to allow the switchoff transient of the photoconductor to be observed. Figure 4 shows typical transient responses observed in the three detector types.

In what follows, the illumination step size is expressed in terms of the change in the carrier

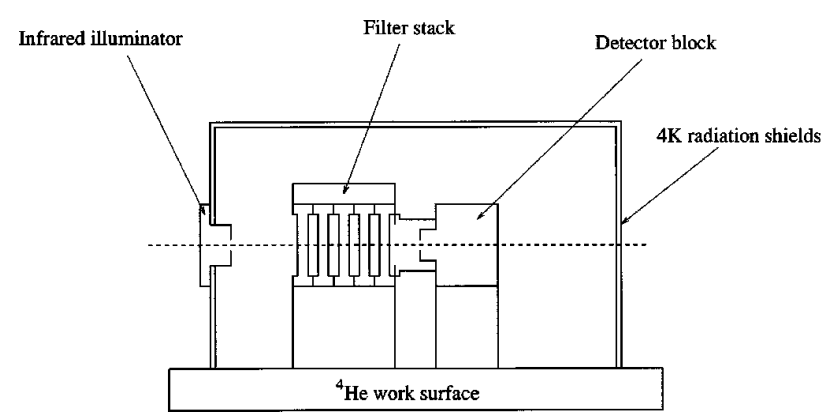

Fig. 3. Experimental configuration used to measure the detector response to a step change in illumination. 


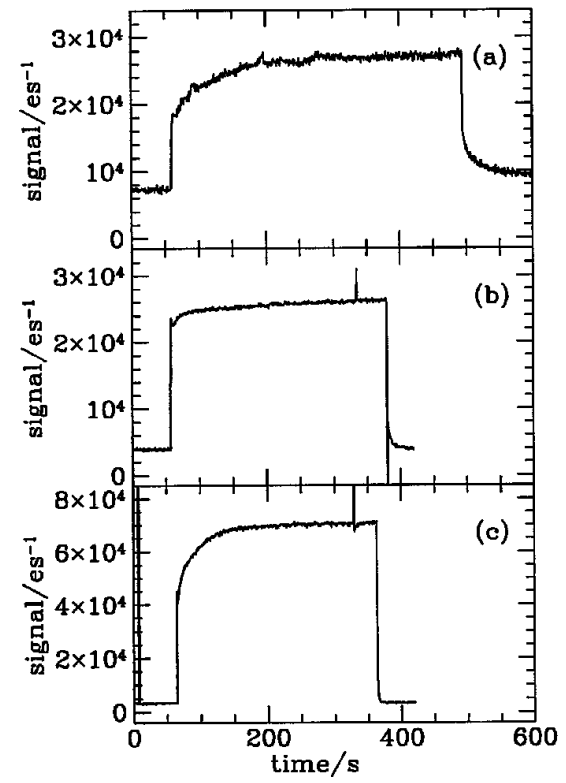

Fig. 4. Typical response curves to a step in illumination of (a) a Ge:Be detector operated at $3 \mathrm{~K}, 50 \mu \mathrm{m}$, with a bias field of $6 \mathrm{~V}$ $\mathrm{cm}^{-1}$; (b) a Ge:Ga $(u)$ detector operated at $3 \mathrm{~K}, 85 \mu \mathrm{m}$, with a bias field of $2 \mathrm{~V} \mathrm{~cm}^{-1}$; (c) a Ge:Ga $(s)$ detector operated at $1.85 \mathrm{~K}, 160 \mu \mathrm{m}$, with a bias field of $0.6 \mathrm{~V} \mathrm{~cm}^{-1}$. The illumination step was chosen to give a similar value of $\Delta g\left(\sim 2 \times 10^{8} \mathrm{~cm}^{-3} \mathrm{~s}^{-1}\right)$ in each case.

generation rate, $\Delta g=\left(\Delta I_{\text {det }} / V_{c} G\right) \mathrm{cm}^{-3} \mathrm{~s}^{-1}$, where $G$ is the photoconductive gain, $V_{c}$ is the volume of the detector crystal in cubic centimeters, and $I_{\mathrm{det}}$ is the detector current in electrons per second. The photoconductive gain is calculated from measurements of the responsivity and quantum efficiency of the detectors. Typical values of $G$ (which is a function of bias voltage) for the LWS detectors range from 0.1 for $\mathrm{Ge}: \mathrm{Be}\left(E_{b}=6 \mathrm{~V} \mathrm{~cm}^{-1}\right)$ and Ge:Ga $(u)\left(E_{b}=2 \mathrm{~V} \mathrm{~cm}^{-1}\right)$ to 0.3 for $\mathrm{Ge}: \mathrm{Ga}(s)\left(E_{b}=0.7 \mathrm{~V} \mathrm{~cm}^{-1}\right)$.

\section{A. Transient Response in a Ge:Ga $(u)$ Detector}

The transient response of a LWS Ge:Ga $(u)$ detector as a function of illumination step size, $\Delta g$, and bias electric field, $E_{b}$, was measured at $3 \mathrm{~K}$ and $85 \mu \mathrm{m}$. Measurements were made at four different bias levels, keeping $\Delta g$ fixed, and then at five different values of $\Delta g$, keeping $E_{b}$ fixed. It was found that after a step change in illumination, the time variation of the detector current, $I_{\mathrm{det}}$, can always be well fitted by an empirical model containing three time constants (see Fig. 5):

$$
\begin{aligned}
I_{\operatorname{det}}= & I_{0}\left[1+a_{1} \exp \left(-t / \tau_{1}\right)+a_{2} \exp \left(-t / \tau_{2}\right)\right. \\
& \left.+a_{3} \exp \left(-t / \tau_{3}\right)\right] .
\end{aligned}
$$

where $I_{0}$ is the final settled value of the current. The amplitudes, $a_{i}$, and time constants, $\tau_{i}$, are functions of the specific operating conditions. The combination of the terms in $\tau_{1}$ and $\tau_{2}$ generate the characteristic pulse-hook response (see Figs. 1, 2, and 4) if $a_{1}$ is positive and $a_{2}$ is negative. The LWS $\mathrm{Ge}: \mathrm{Ga}(u)$ detectors always exhibit a pulse-hook re-

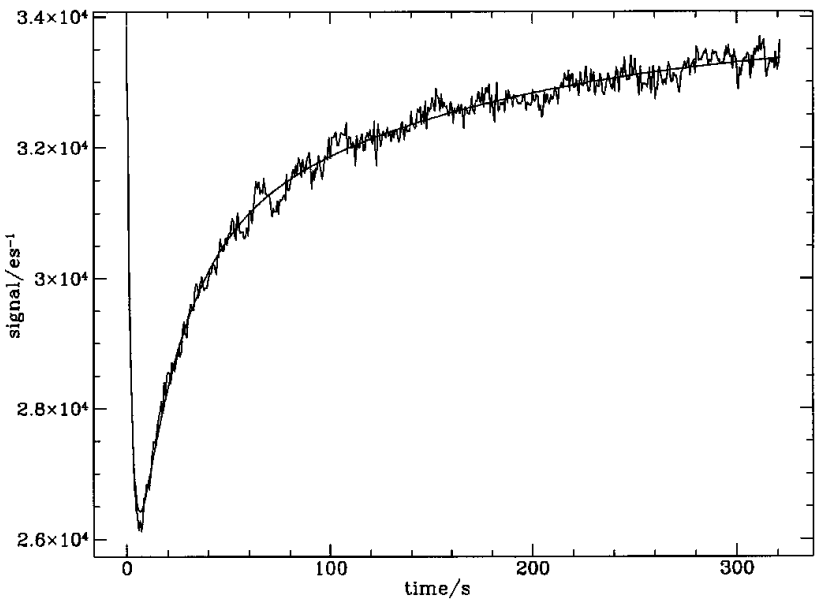

Fig. 5. Results of a three time-constant fit to the step response of the $\mathrm{Ge}: \mathrm{Ga}(u)$ detector operated at $3 \mathrm{~K}, 85 \mu \mathrm{m}$ and a bias field of $2 \mathrm{~V}$ $\mathrm{cm}^{-1}$. The smooth curve is the best fit of the model to the data.

sponse to a step illumination change under conditions similar to those expected in flight. The third time constant, $\tau_{3}$, in this empirical model dominates the overall settling time.

Figure 6 illustrates the dependence of $\tau_{i}$ on $\Delta g$ and $E_{b}$. Also shown is the time taken for the signal to settle to $1 \%$ of its final value $\left(t_{1 \%}\right)$, calculated from the empirical model with the appropriate fitted parameters. Because the settling times are much stronger functions of bias than of illumination step size, they must be a property of the detector, not the illuminator.

When the bias is fixed, $\tau_{2}$ and $\tau_{3}$ are virtually independent of $\Delta g$ while the fastest time constant, $\tau_{1}$, does show a slight negative dependence, decreasing by a factor of 2 if $\Delta g$ is increased by a factor of 4 .

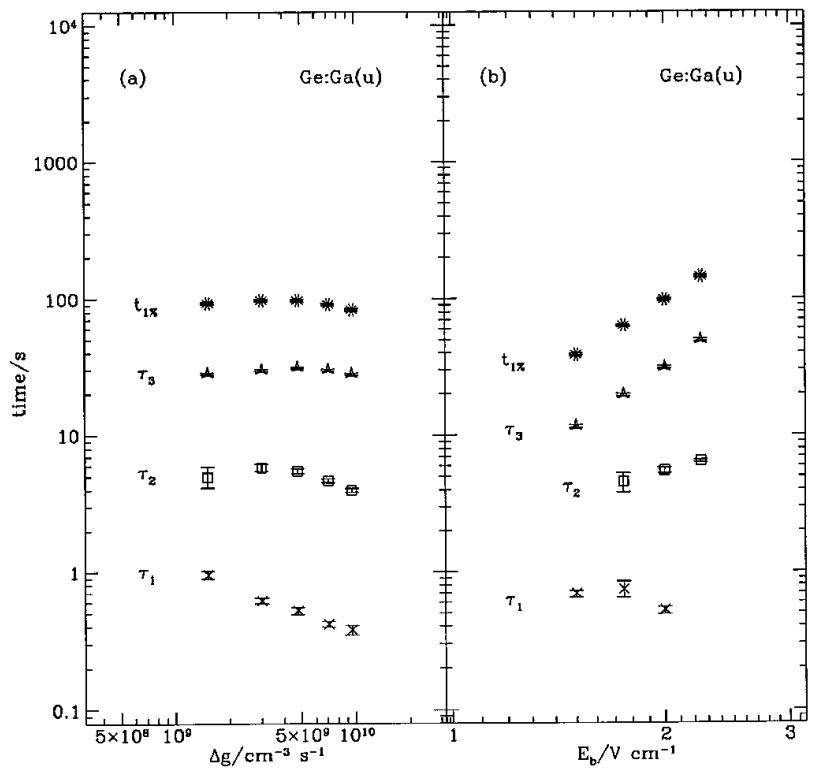

Fig. 6. Dependence of fitted time constants on (a) illumination step size at a fixed bias of $2 \mathrm{~V} \mathrm{~cm}^{-1}$ and (b) bias at a fixed illumination step of $5 \times 10^{9} \mathrm{~cm}^{-3} \mathrm{~s}^{-1}$ for the Ge:Ga $(u)$ detector. The operating temperature was $3 \mathrm{~K}$. 
When the illumination step size is fixed, $\tau_{1}$ decreases slightly with increasing bias while $\tau_{2}$ and $\tau_{3}$ show a strong positive dependence on $E_{b}$. Consequently, the hook response is faster at higher bias and signal levels. The overall settling time is almost independent of flux step size, but increases as $E_{b}^{3.3}$. Thus the long settling time of the detector can be reduced by operating at a lower bias, but at the cost of decreased responsivity and sensitivity.

\section{B. Transient Response in a Ge:Ga(s) Detector}

The LWS Ge:Ga $(s)$ detectors are identical to the $\mathrm{Ge}: \mathrm{Ga}(u)$ detectors, apart from the application of stress to the crystal to increase the long wavelength cutoff. The transient response of a LWS Ge: $\mathrm{Ga}(s)$ detector was measured at $1.85 \mathrm{~K}$ and $160 \mu \mathrm{m}$. Figure 4 illustrates how the behavior of this detector differs from that of its unstressed counterpart. A pulse-hook response is visible on the transient response of the Ge: $\mathrm{Ga}(s)$ detector, but it is clearly very fast $(\ll 1 \mathrm{~s})$. This is in contrast to the Ge:Ga $u$ detector, which shows a slow pulse-hook response that becomes slower still as the operating temperature is reduced (see Fig. 2). The three time-constant empirical model also provides good fits to the $\mathrm{Ge}: \mathrm{Ga}(s)$ step response curves. Figure 7 shows the variation of $\tau_{2}$ and $\tau_{3}$ with $\Delta g$ and $E_{b}\left(\tau_{1}\right.$ is $\ll 0.2 \mathrm{~s}$ and thus is too fast to be accurately measured with this experimental arrangement). At fixed bias, $t_{1 \%}$ shows a weak negative dependence on $\Delta g$, decreasing by a factor of 3 with a factor of 10 increase in the flux step. This is in contrast to the lack of dependence of $t_{1 \%}$ on $\Delta g$ exhibited by the Ge:Ga $(u)$ detector. The bias dependence of $t_{1 \%}$ is also weak, scaling as $E_{b}^{0.6}$ compared with $E_{b}^{3.3}$ for the Ge:Ga $(u)$ device.

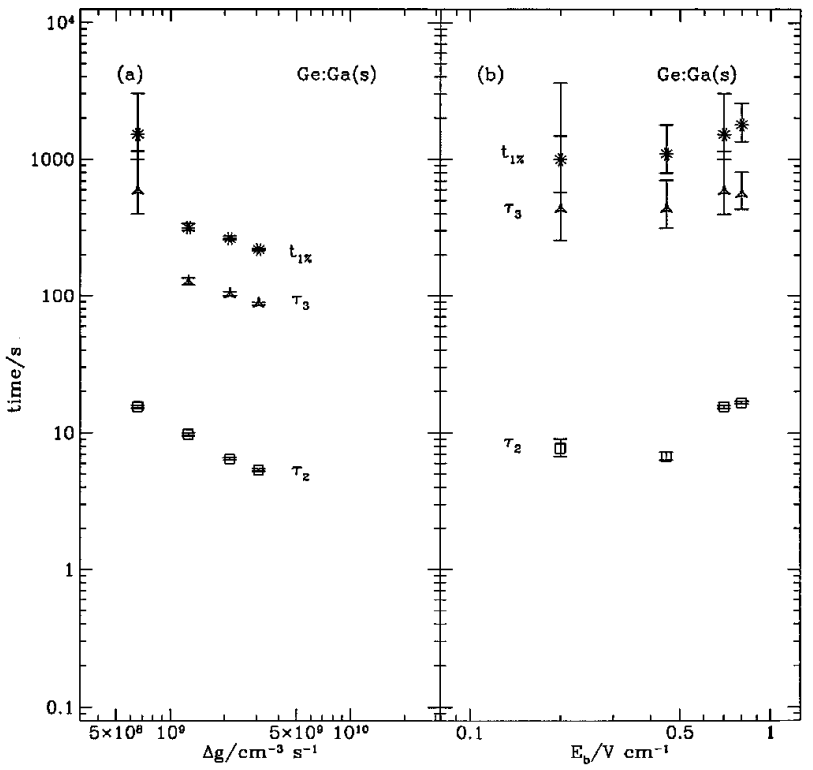

Fig. 7. Dependence of fitted time constants on (a) illumination step size at a fixed bias of $0.7 \mathrm{~V} \mathrm{~cm}^{-1}$ and (b) bias at a fixed illumination step of $7 \times 10^{8} \mathrm{~cm}^{-3} \mathrm{~s}^{-1}$ for the Ge:Ga(s) detector. The operating temperature was $1.85 \mathrm{~K}$.

\section{Transient Response of a Ge:Be Detector}

The transient behavior of a LWS Ge:Be detector to step changes in illumination has been investigated at $3 \mathrm{~K}$ and $50 \mu \mathrm{m}$. The crystal size and mounting configuration are identical to that of the $\mathrm{Ge}: \mathrm{Ga}(u)$ detector. The transient response curves can also be fitted by the three time-constant model for all values of $E_{b}$ and $\Delta g$. However, there are some important differences between the behavior of this detector and that of the Ge:Ga detectors. First, the $a_{i}$ parameters all take positive values; consequently, there is no pulse-hook response (see Fig. 4). Second, a noticeably long switch-off transient can also be fitted by the three time-constant model, but with time constants that are always shorter than the corresponding switch-on constants. The switch-off transient seen in Ge:Ga detectors is extremely short compared with that of the Ge:Be detector.

Figure 8 shows the variation of the $\tau_{i}$ values fitted to the switch-on transient as a function of $\Delta g$ and $E_{b}$. The time constants show a negative dependence on $\Delta g$ comparable with that observed in the $\mathrm{Ge}: \mathrm{Ga}(s)$ detectors. The $1 \%$ settling time decreases approximately linearly with increasing $\Delta g$ but depends only weakly on bias.

\section{Effects of Operating Temperature on the Transient} Response Times of the Long Wavelength Spectrometer Unstressed Detectors

The effect of operating temperature on the transient response times of both the LWS Ge:Ga $(u)$ and Ge:Be detectors has also been investigated. The experimental configuration used in this study is unsuitable for measuring the transient response of $\mathrm{Ge}: \mathrm{Ga}(s)$ at temperatures above $1.85 \mathrm{~K}$ because the dark current

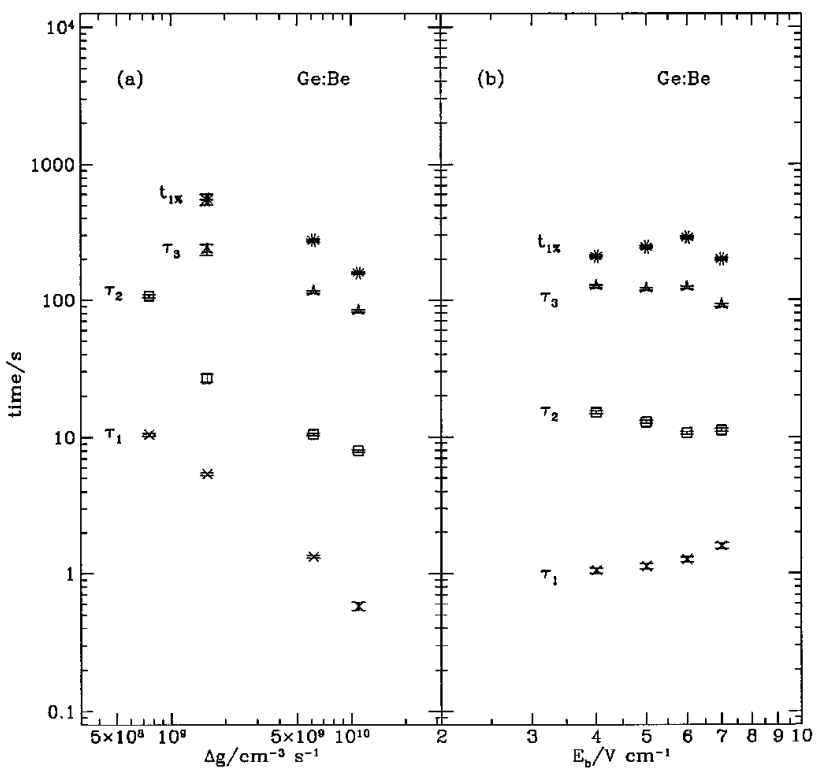

Fig. 8. Dependence of fitted time constants on (a) illumination step size at a fixed bias of $6 \mathrm{~V} \mathrm{~cm}^{-1}$ and (b) bias at a fixed illumination step of $6 \times 10^{9} \mathrm{~cm}^{-3} \mathrm{~s}^{-1}$ for the Ge:Be detector. The operating temperature was $3 \mathrm{~K}$. 
increases strongly with increased temperature and quickly saturates the integrating amplifier.

Figure 9 shows the variation of $\tau_{i}$ with temperature for the two detector types. The $1 \%$ settling time of the $\mathrm{Ge}: \mathrm{Ga}(u)$ detector decreases as roughly the 20th power of the temperature. Consequently, by operation of these detectors at $3 \mathrm{~K}$ in the LWS, the response time, as well as freedom from spontaneous spiking (see Subsection 1.B.), is considerably improved. In contrast, the Ge:Be detector shows a weak increase in response time with increased temperature. However, by operation of this detector at $3 \mathrm{~K}$ in the LWS rather than at $2.5 \mathrm{~K}$, spontaneous spiking is reduced at the cost of an increase in response time of only $30 \%$.

For the differences in settling behavior observed in the three LWS detector types to be summarized, power laws have been fitted to the $1 \%$ settling times shown in Figs. 6-9. Clearly, not all the data are well fitted by a power law; however, the indices summarized in Table 2 serve as a rough guide to the behavior of each detector type and allow the different devices to be compared to first order.

\section{Modeling of Inherent Nonlinear Effects}

The transient response of an extrinsic photoconductor to a change in incident photon flux has been addressed theoretically with varying degrees of complexity and assumptions. In its simplest form, the transient response is determined by the lifetime of the excess carriers. For extrinsic Ge photoconductors operated at low photon backgrounds, this lifetime is determined by the concentration of minority dopants, $N_{D}$ (which produce the ionized sites for recombination), and the product of capture cross section, $\sigma$, and carrier velocity, $v$, such that $\tau=$

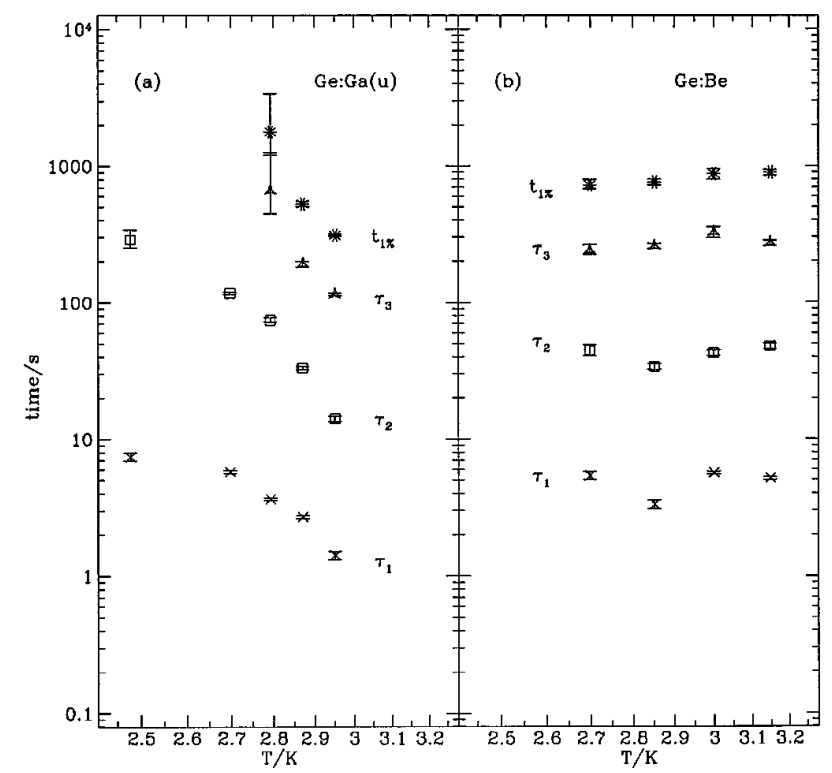

Fig. 9. Dependence of fitted time constants on detector operating temperature for (a) a Ge: $\mathrm{Ga}(u)$ detector at a fixed bias of $2 \mathrm{~V} \mathrm{~cm}-1$ and a step size of $5 \times 10^{8} \mathrm{~cm}^{-3} \mathrm{~s}^{-1}$ and (b) a Ge:Be detector at a fixed bias of $6 \mathrm{~V} \mathrm{~cm}^{-1}$ and a fixed step size of $10^{9} \mathrm{~cm}^{-3} \mathrm{~s}^{-1}$.
Table 2. Summary of the Power Law Indices Determined for $t_{1 \%}$ as a Function of $\Delta g, E_{b}$, and $T$ from Figs. 6-9

\begin{tabular}{lccc}
\hline Parameter & $\mathrm{Ge}: \mathrm{Ga}(u)$ & $\mathrm{Ge}: \mathrm{Ga}(s)$ & $\mathrm{Ge}: \mathrm{Be}$ \\
\hline$\Delta g\left(\mathrm{~cm}^{-3} \mathrm{~s}^{-1}\right)$ & $-0.01 \pm 0.02$ & $-0.45 \pm 0.07$ & $-0.96 \pm 0.06$ \\
$E_{b}\left(\mathrm{~V} \mathrm{~cm}^{-1}\right)$ & $3.30 \pm 0.13$ & $0.60 \pm 0.76$ & $-0.57 \pm 0.10$ \\
$T(\mathrm{~K})$ & $-19.5 \pm 1.8$ & - & $1.6 \pm 0.7$ \\
\hline
\end{tabular}

$(N \sigma v)^{-1}$. For commonly used materials, with detector operating temperatures of $2-4 \mathrm{~K}$, this time is of the order of $10^{-9}-10^{-7} \mathrm{~s}$.

However, this model is valid only for an infinitely long detector with no additional trapping centers. In practice, the transient response can be dominated either by trapping phenomena ${ }^{3,12}$ or by carrier sweep out, out diffusion, or both, which are associated with the finite length of the device. ${ }^{13,14}$ We compare the experimental results obtained here to current models.

It has long been known that defect centers shallower than the primary dopant can lead to trapping. The high-quality materials used for Ge:Ga and Ge:Be photoconductors are generally not affected by unintended dopants, but in 1989 Haegel et al. showed that the trapping associated with $\mathrm{Be}^{+}$centers can explain slow transient response in Ge:Be detectors. ${ }^{3}$ Nonequilibrium $\mathrm{Be}^{+}$centers form when a neutral $\mathrm{Be}$ center captures an additional hole, ${ }^{15}$ analogous to the formation of $\mathrm{D}^{-}$centers in $n$-type semiconductors. One candidate, therefore, for the slow transient response of the LWS Ge:Be and Ge:Ga detectors is the trapping associated with $\mathrm{Be}^{+}$and $\mathrm{Ga}^{+}$centers.

Transient effects caused by trapping are strongly dependent on temperature because the time required to equilibrate the traps depends exponentially on the ratio of trap energy to thermal energy, $k T$. By taking into account the temperature dependence of the free carrier lifetime and the density of states, one can estimate the energy level of the trap involved. ${ }^{3}$ The LWS Ge:Be detectors exhibit a settling time that actually increases with increasing temperature, thus ruling out any type of dominant thermal trapping mechanism. For the Ge:Ga $(u)$ devices, the temperature dependence of $\tau_{3}$ is much steeper (corresponding to an activation energy $\epsilon_{\exp }=6$ meV) than is predicted ${ }^{16}$ for $\mathrm{Ga}^{+}$trapping $\left(\epsilon_{\mathrm{Ga}^{+}}=2.4\right.$ $\mathrm{meV}$ ). We conclude that, for the LWS unstressed detectors at least, trapping is not the primary cause of the slow transient responses we observe.

The second phenomenon that can lead to long transients in extrinsic photoconductors is the sweep out of carriers after an increase in photon flux. This leads to a period of time during which excess space charge forms inside the device, producing a change in the electric field near the contact that counteracts the sweep out of the carriers, allowing the device to reach its new equilibrium level of current flow. This behavior occurs primarily under very low background conditions and relatively high levels of applied bias, and it has been modeled analytically by Fouks $^{13}$ and numerically by Haegel et al. ${ }^{14}$ Initial 
research by Haegel et al. on thin detectors at low bias (where out diffusion is dominant) has recently been extended to standard size detectors under actual operating biases.

In the small signal numerical analysis of Haegel et $a l$., the transient response is found to consist of both a fast and a slow component. The time constant of the fast component is given by the free carrier lifetime, whereas the slow component cannot be described with a single exponential time constant. This is consistent with the empirical fitting of multiple time constants used in Section 3. This model also produces a pulse-hook effect like that observed in the LWS, and other, extrinsic photoconductors.

The model predicts a slow transient response that is inversely dependent on the hole-generation rate, implying that the transient time can vary from temperature independent (or even weakly increasing) when the generation is completely photon dominated, to strongly decreasing $(\tau$ becomes much shorter with increasing temperature) when thermal generation begins to play a significant role. Both types of behavior have been observed in the LWS detectors.

The electric-field dependence in the Haegel et al. model has two effects. First, the magnitude of the slow component increases with increased $E_{b}$ as additional carriers are initially swept out of the device. Second, when sweep out is dominant, the slow transient time is, to first order, linear in the applied field $E_{b}$. Thus the effect of field on both net generation rate and the magnitude of the slow component can be combined to obtain a variety of results for the dependence of a fitted parameter such as $\tau_{3}$. The variations with detector material are most probably associated with different optical versus thermal generation rates, as well as field effects, for the different dopant ionization energies.

It is difficult to compare the actual detector results with predictions from the numerical model because the model is based on a small signal analysis, i.e., changes in photon flux that are small compared with the initial background flux. The experiments, in contrast, are conducted in a large signal limit, where the change in flux is many orders of magnitude higher than the initial value. A typical example would be a starting carrier generation rate of $10^{5}$ $\mathrm{cm}^{-3} \mathrm{~s}^{-1}$, followed by the addition of a signal with an optical generation rate of $10^{9} \mathrm{~cm}^{-3} \mathrm{~s}^{-1}$. If we consider the results of the small-signal numerical modeling for Ge:Ga detectors under comparable bias, we find transient times ranging from $0.1 \mathrm{~s}$ (for a background of $10^{9} \mathrm{~cm}^{-3} \mathrm{~s}^{-1}$ ) to $1000 \mathrm{~s}$ (for a background of $10^{5} \mathrm{~cm}^{-3} \mathrm{~s}^{-1}$ ), bracketing the experimental transient times observed. Although the model predicts a transient time that is inversely proportional to the generation rate, the weaker flux dependence observed here may indicate a saturation of this effect at very large flux steps.

The analytical model developed by Fouks can be applied in a large signal limit. This approach, however, requires the assumption that internal field changes are small compared with the external applied field. Although this is often a good assumption for Si photoconductors, where relatively large applied fields can be used, it is less valid for Ge, where shallower levels result in lower breakdown fields. Like the numerical model, the Fouks model predicts an inverse dependence on photon flux, as well as a dependence on device gain.

To summarize, a qualitative analysis of the transient data obtained for the LWS Ge photoconductors indicates that carrier sweep out followed by electricfield adjustments in the near-contact region is the most probable cause of the long transient response. Numerical modeling in the small-signal limit shows that this phenomenon can lead to transient times of the order of magnitude of those observed. For these models to be used to predict actual settling times, however, additional numerical modeling is required to address the effect of the large step size in photon flux. Algorithms could be developed, based on these models, to allow predictions of final current levels based on initial transient behavior.

\section{Conclusions}

We have explored transient effects as a function of detector type, bias, and operating temperture. Spontaneous spiking causes significant degradation of the performance of the Ge:Be and Ge:Ga $(u)$ detectors at temperatures below $2.5 \mathrm{~K}$, whereas the $\mathrm{Ge}: \mathrm{Ga}(s)$ detectors remain well behaved even below $1.8 \mathrm{~K}$. The effects of bias and illumination step size on the slow relaxation after an illumination change have been systematically investigated, and a simple empirical model for the time response has been developed. There are significant differences in behavior among the three detector types.

The results have been compared with models for characteristic settling times. Although a single characteristic time may be successful in reproducing some features of observed behavior, very complicated modeling, requiring numerical integration with boundary conditions specific to the particular operating conditions, will be required to reproduce accurately the transient response curves. For practical applications, such as improved calibration of the LWS, empirical modeling of the type presented here may be more appropriate.

Inherent nonlinear effects are not expected to pose a problem during LWS observations of astronomical sources in which a diffraction grating or a diffraction grating and Fabry-Perot interferometer combination is moved to scan the spectrum of the source across the detectors. Laboratory tests of the assembled instrument have shown that the smallsignal changes that generally occur do not significantly perturb the detector and that the response is linear. Transient response will affect calibration with the on-board illumination sources but may be reduced by employment of frequent calibration with a gradual increase rather than a large step change in signal. The actual calibration scheme chosen and 
the frequency of calibrations will take into account changes in behavior that may be induced by the ionizing radiation environment encountered in orbit. ${ }^{17}$

Spontaneous spiking is prevented by operation of the unstressed detectors at $3 \mathrm{~K}$, where these problems do not occur. Additionally, if unexpected spiking behavior should be encountered in flight, the ability exists to reduce the bias level of each detector independently so that spontaneous breakdown will not occur. Through careful operation of the LWS, there should be a minimal loss in total observation time caused by these nonlinear effects.

This research was supported by Science and Engineering Research Council grant SGC24450. M. Price acknowledges receipt of a Science and Engineering Research Council studentship.

\section{References and Notes}

1. P. R. Bratt, "Impurity germanium and silicon infrared detectors," Semicond. Semimet. 12, 39-142 (1977).

2. S. W. Teitsworth, R. M. Westervelt, and E. E. Haller, "Nonlinear oscillations and chaos in electrical breakdown in Ge," Phys. Rev. Lett. 51, 825-828 (1983).

3. N. M. Haegel, J. W. Beeman, P. N. Luke, and E. E. Haller, "Transient photoconductivity in Ge:Be due to $\mathrm{Be}^{+}$formation," Phys. Rev. B 39, 3677-3682 (1989).

4. N. M. Haegel and E. E. Haller, "Transient response of Ge:Be and Ge:Zn far-infrared photoconductors under low background photon flux conditions," Infrared Phys. 26, 247-261 (1986).

5. N. Sclar, "Properties of doped silicon and germanium infrared detectors," Prog. Quantum Electron. 9, 149-257 (1984).

6. J. W. Wensink, W. Luinge, D. Beintema, E. A. Valentijn, T. de Graauw, R. Katterloher, L. Barl, and E. T. Young, "Characteristics of the ISO Short Wavelength Spectrometer detec- tors," in Proceedings of the European Space Agency Symposium on Photon Detectors for Space Instrumentation (ESA, Nordwijk, 1992), SP-356, pp. 339-344.

7. Battelle-Institut e.V., Am Römerhof 35, D-6000 Frankfurt a.M. 90, Germany.

8. M. Overhamm, Battelle-Institut e.V., Am Römerhof 35, D-6000 Frankfurt a.M. 90, Germany (personal communication, 1992).

9. Model TRS, Infrared Labs, Tucson, Ariz. 85719.

10. S. E. Church, M. J. Griffin, P. A. R. Ade, M. C. Price, R. J. Emery, and B. M. Swinyard, "Calibration and performance testing of doped-germanium photoconductors for the ISO Long Wavelength Spectrometer," Infrared Phys. 34, 389-406 (1993).

11. Model JF4, Infrared Labs, Tucson, Ariz. 85719.

12. S. M. Ryvkin, Photoelectric Effects in Semiconductors (Consultants Bureau, New York, 1964).

13. B. I. Fouks, "Non-stationary behavior of low background photon detectors," in Proceedings of the ESA Symposium on Photon Detectors for Space Instrumentation (European Space Agency, Noordwijk, 1992), SP-356, pp. 167-174, and references therein.

14. N. M. Haegel, C. A. Latasa, and A. M. White, "Transient response of infrared photoconductors: the roles of contacts and space charge," Appl. Phys. A 56, 15-21 (1993).

15. E. E. Haller, R. E. MacMurray, Jr., L. M. Falicov, N. M. Haegel, and W. L. Hansen, "Three holes bound to a double acceptor: $\mathrm{Be}^{+}$in germanium," Phys. Rev. Lett. 51, 10891091 (1983).

16. E. I. Gershenzon, G. N. Gol'tsman, A. P. Mel'nikov, "Binding energy of a carrier with a neutral impurity atom in germanium and in silicon," Pis'ma Zh. Eksp. Teor. Fiz. 14, 281-282 (1971) [JETP Lett. 14, 185-186 (1971)].

17. M. C. Price, M. J. Griffin, S. E. Church, A. G. Murray, and P. A. R. Ade, "Ionising radiation induced effects in doped germanium FIR photoconductors," in Proceedings of the European Space Agency Symposium on Photon Detectors for Space Instrumentation (ESA, Nordwijk, 1992), SP-356, pp. 309-312. 